\title{
Tunable narrow-band terahertz generation from periodically poled lithium niobate
}

\author{
Y. S. Lee, ${ }^{\text {a) }}$ T. Meade, and T. B. Norris \\ Center for Ultrafast Optical Science, The University of Michigan, Ann Arbor, Michigan 48109-2099
}

A. Galvanauskas

IMRA America, 1044 Woodridge Avenue, Ann Arbor, Michigan 48105

(Received 14 February 2001; accepted for publication 27 March 2001)

\begin{abstract}
We describe a technique for generating tunable narrow-band terahertz radiation via optical rectification in periodically-poled lithium niobate. Frequency tuning is accomplished by spatially chirping the domain width laterally to the beam propagation direction, and adjusting the temperature of the sample. We demonstrate tuning over a continuous range from 0.8 to $2.5 \mathrm{THz}$. The bandwidth of the terahertz waveforms is as narrow as $0.02 \mathrm{THz}$. (C) 2001 American Institute of Physics.
\end{abstract}

[DOI: $10.1063 / 1.1373406$ ]

There is presently considerable research activity in the development of the new sources in the terahertz (THz) region of the spectrum. The recent development of femtosecond lasers naturally brought about various techniques to generate broad-band, single-cycle $\mathrm{THz}$ waves, since the inverse pulse duration of femtosecond lasers corresponds to the $\mathrm{THz}$ frequency range. These sources have found many applications, such as THz spectroscopy for studies of carrier dynamics and intermolecular dynamics in liquids, ${ }^{1,2}$ dielectric response of molecules, polymers and semiconductors, ${ }^{3-5}$ etc. Since single-cycle waves intrinsically possess broad bandwidth, they are particularly useful for investigating the $\mathrm{THz}$ response of matter over a wide range of spectrum concurrently.

In many cases, on the other hand, spectrally narrow and bright $\mathrm{THz}$ sources are desired when the phenomenon under investigation has relatively narrow bandwidth. Although many ways of generating single-cycle $\mathrm{THz}$ waves with broad bandwidth have been developed, few schemes exist to generate narrow-band $\mathrm{THz}$ waves. One promising technique for generating narrow-band $\mathrm{THz}$ waves has been demonstrated, using optical rectification of femtosecond pulses in periodically poled lithium niobate (PPLN) ${ }^{6,7}$ Narrow-band sources are in great demand for a wide range of applications in spectroscopy, sensing, communication, and imaging; for such applications frequency tunability is essential. In this letter, we demonstrate the frequency tuning of narrow-band $\mathrm{THz}$ radiation from PPLN crystals by lateral spatial chriping of the domain width and adjusting the temperature of the samples.

In the experiment, we use two types of $z$-cut PPLN crystals which were laterally chirped, i.e., multiple domain structures of slightly different domain width were fabricated side by side at a regular distance from one another. Sample 1 is 6 $\mathrm{mm}$ long and has seven domain structures. The domain width varies from 30 to $60 \mu \mathrm{m}$ with a $5 \mu \mathrm{m}$ step size. Sample 2 is $10 \mathrm{~mm}$ long and has 46 domain structures. The domain width varies from 21.5 to $62 \mu \mathrm{m}$ with a $0.9 \mu \mathrm{m}$ step size. Tuning of the $\mathrm{THz}$ is accomplished simply by scanning the sample laterally to the beam propagation direction; fine tuning is ac-

${ }^{a)}$ Electronic mail: yunslee@eecs.umich.edu complished via temperature tuning, as will be discussed later.

Narrow-band THz wave forms are generated via optical rectification of pump pulses generated from a $250 \mathrm{kHz}$ Ti:Sapphire regenerative amplifier. ${ }^{8}$ The pump spectrum is centered at $800 \mathrm{~nm}$ and the pulse duration is $200 \mathrm{fs}$. Pump pulses of $50 \mathrm{~nJ}$ are focused to a $100 \mu \mathrm{m}$ spot on the samples. The samples are placed in a cryostat in which the temperature of the sample can be varied continuously between 10 and $300 \mathrm{~K}$. The generated $\mathrm{THz}$ waves are focused by a pair of off-axis parabolodial mirrors into a $1 \mathrm{~mm}$ (110)-cut ZnTe crystal. The $\mathrm{THz}$ wave form is measured by free-space electro-optic sampling. ${ }^{9}$

As the femtosecond optical pulses propagate through the PPLN crystal, a THz nonlinear polarization is generated via optical rectification. Each domain in the PPLN contributes a half cycle to the radiated $\mathrm{THz}$ field due to the walk off between the optical and THz pulses. ${ }^{6,7}$ In the absence of absorption and domain-width fluctuations, the relative bandwidth $\Delta \nu / \nu$ of the THz field is given simply as $2 / N$, where $N$ is the number of domains in the PPLN. The frequency of the $\mathrm{THz}$ wave is determined as

$$
\nu_{T}=c / 2 d\left(n_{T}-n_{O}\right),
$$

where $c$ is the light velocity, $d$ is the domain width, and $n_{T}\left(n_{O}\right)$ is the group refractive index at $\mathrm{THz}$ (optical) frequency. ${ }^{6}$ Thus, frequency tuning can be accomplished by adjusting the domain width of the PPLN. Figures 1(a) and 1(b) show the $\mathrm{THz}$ wave forms and the respective spectra from the sample 1 when the domain width is $30,40,50$, and $60 \mu \mathrm{m}$ at $T=115 \mathrm{~K}$. Figure 1 clearly shows that the $\mathrm{THz}$ wave from the shorter domains is generated with higher frequency as expected from Eq. (1). Some features of the THz wave forms and spectra of Fig. 1 are noteworthy. Because of the material absorption at $\mathrm{THz}$ frequencies, the $\mathrm{THz}$ wave decays as it propagates through the PPLN. The absorption is more severe at higher frequencies, because the main source of the absorption is the low-frequency tail of the transverse optical phonon mode at $7.6 \mathrm{THz}{ }^{10}$

It has been demonstrated that the absorption of the $\mathrm{THz}$ waves in PPLN crystals is suppressed substantially at a low 

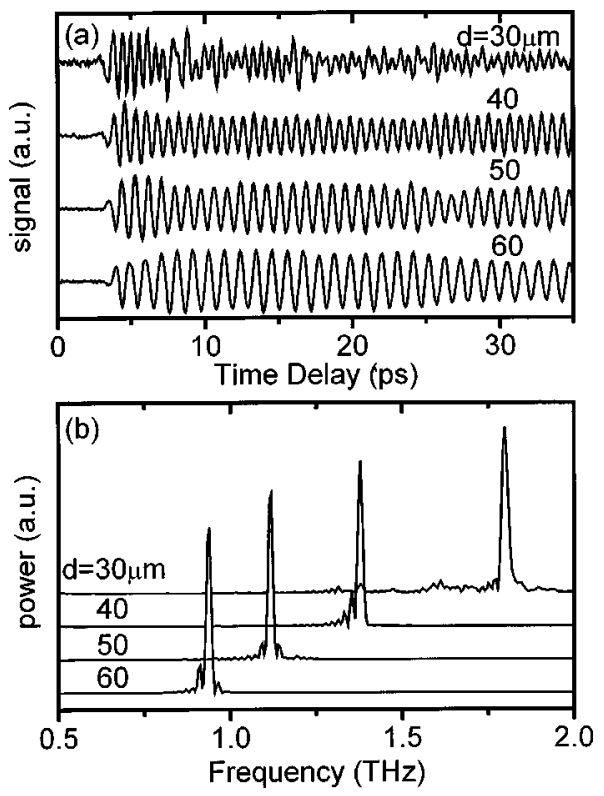

FIG. 1. (a) THz wave forms and (b) power spectra at $T=115 \mathrm{~K}$ when the domain width is $30,40,50$, and $60 \mu \mathrm{m}$.

temperature. ${ }^{7}$ Figure 2 shows the measured $\mathrm{THz}$ wave forms and power spectra at $T=12,60,90,120,150,180,210,240$, and $270 \mathrm{~K}$ from the $35 \mu \mathrm{m}$ domain-width region in sample 1. The temporal decay of the $\mathrm{THz}$ wave form becomes important for temperatures above about $100 \mathrm{~K}$, and the absorption increases gradually as the temperature increases. Due to the absorption, the bandwidth of the $\mathrm{THz}$ emission increases at high temperature. It is also observed that the spectrum shifts to the red with increasing temperature. The spectral shift from 12 to $270 \mathrm{~K}$ is $0.1 \mathrm{THz}$, which corresponds to $6.5 \%$ of the central frequency $(1.55 \mathrm{THz}$ at $T=150 \mathrm{~K})$ of the $\mathrm{THz}$ waves. The frequency shift is due to an interplay of the temperature-dependent index of refraction and the thermal expansion of the sample. Since the thermal expansion coefficient of lithium niobate is $4.1 \times 10^{-6} \mathrm{~K}^{-1}$ in the normal direction of the optic axis, ${ }^{11}$ the change of the domain width from 10 to $270 \mathrm{~K}$ is only $0.1 \%$, which is negligible compared to the spectral shift. Thus, it is clear that the temperature
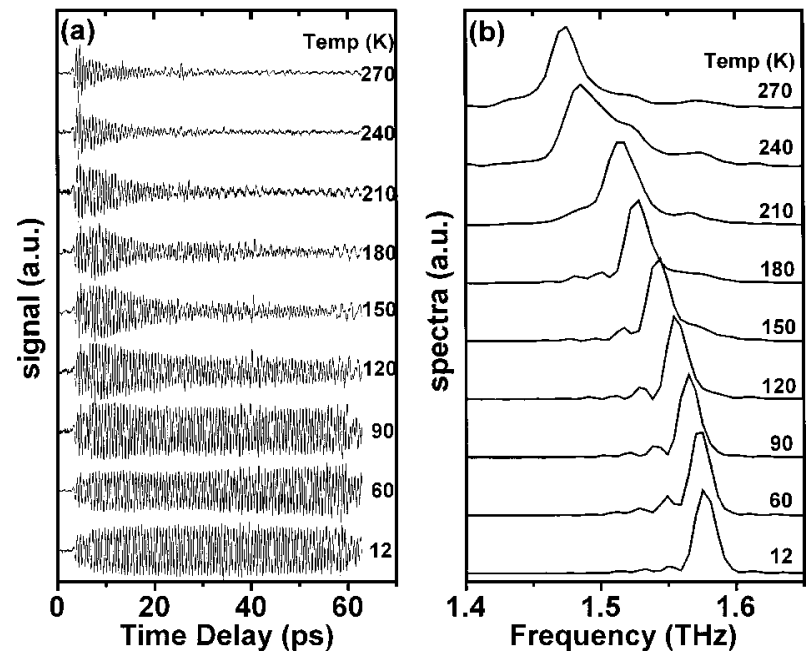

FIG. 2. Measured (a) THz wave forms and (b) spectra at $T=12,60,90,120$, $150,180,210,240$, and $270 \mathrm{~K}$ from the domain structure of $35 \mu \mathrm{m}$ domain width in the $6 \mathrm{~mm}$ sample.
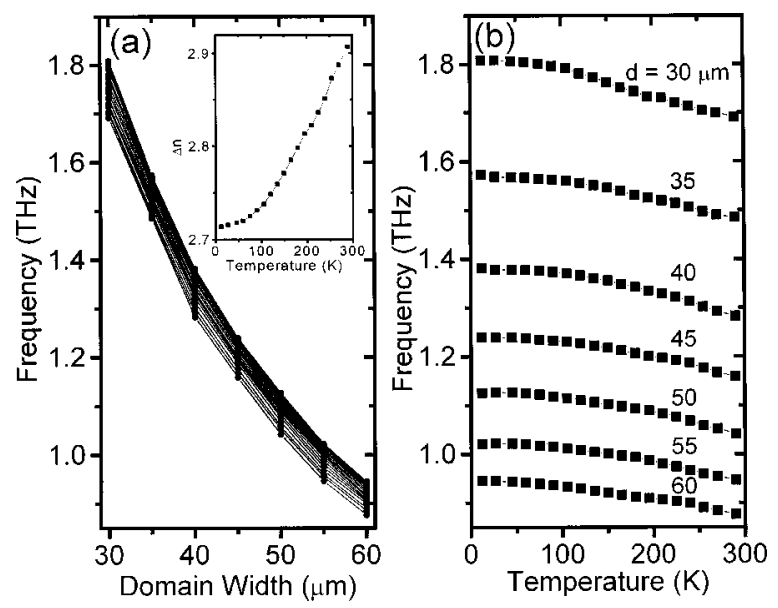

FIG. 3. (a) THz frequency vs the domain width at $T=12,27,45,60,75,90$, $105,120,135,150,165,180,195,210,225,240,255,279$, and $290 \mathrm{~K}$ from top to bottom. $\Delta n$ vs sample temperature is shown in inset. (b) THz frequency vs sample temperature for the domain width of $30,35,40,45,50$, 55 , and $60 \mu \mathrm{m}$.

dependence of the index of refraction at the $\mathrm{THz}$ and the optical frequencies is the dominant factor to explain the spectral shift. Temperature control can be used for the fine tuning of the frequency, though it has some disadvantage due to the wave form distortion by the temperature-dependent absorption.

Figure 3(a) shows the $\mathrm{THz}$ frequency versus the domain width at various sample temperatures for sample 1 . The frequency is inversely proportional to the domain width for a given temperature, and shifts to the red with increasing temperature. Assuming that the thermal expansion of the sample is negligible, $\Delta n\left(=n_{T}-n_{O}\right)$ can be obtained to fit the data using Eq. (1). The fitting results are shown in the inset of Fig. 3(a), which demonstrate the increase of $\Delta n$ with increasing temperature. The fitting result at room temperature $(\Delta n$ $=2.907$ ) agrees well with the reported values of the optical and THz index of refraction, $n_{O}=2.3$ and $n_{T}=5.2 .^{12} \mathrm{In}$ Fig. $3(\mathrm{~b})$, the temperature dependence of the $\mathrm{THz}$ frequency is shown for several domain widths. For the domain widths of 30 and $60 \mu \mathrm{m}$, temperature tuning covers spectral ranges of 0.14 and $0.08 \mathrm{THz}$, respectively.

Fine tuning of the frequency has been performed using sample 2. Figure 4 shows the measured (solid square) and the calculated (solid line) frequency of the $\mathrm{THz}$ wave forms

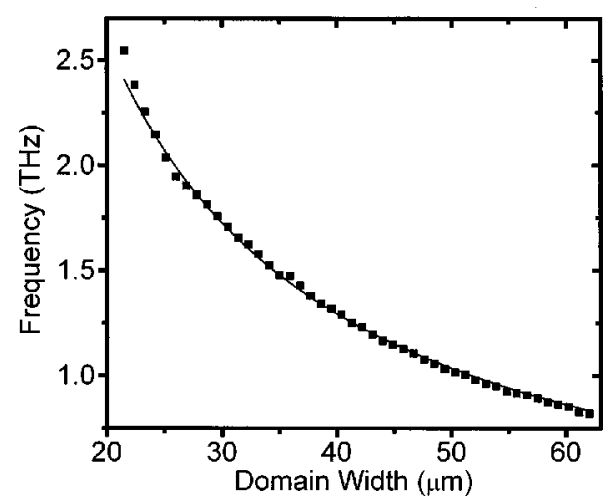

FIG. 4. Measured (solid square) and calculated (solid line) frequency of the $\mathrm{THz}$ waves as a function of domain width using a $10 \mathrm{~mm} z$-cut PPLN at room temperature. 
as a function of the domain width at room temperature. $\Delta n(=2.9)$ at room temperature has been used in the calculation of Eq. (1) to obtain the $\mathrm{THz}$ frequency. There is excellent agreement between the experiment and the calculation, which indicates that the $\mathrm{THz}$ index of refraction is nearly constant within this frequency range. The smaller step size of the domain width in sample 2 as compared to that in sample 1 results in the smaller increment of the $\mathrm{THz}$ frequency with decreasing domain width. The increment of the frequency with $0.9 \mu \mathrm{m}$ step size of the sample 2 is $0.051(0.013) \mathrm{THz}$ for the domain width of $30(60) \mu \mathrm{m}$. Since the temperature tuning range is much bigger than the frequency increment from the domain width change, continuous frequency tuning from 0.8 to $2.5 \mathrm{THz}$ can be accomplished from sample 2 by varying the sample temperature.

In conclusion, we have demonstrated frequency tuning of narrow-band $\mathrm{THz}$ waves from PPLN crystals. The $\mathrm{THz}$ frequency can be tuned continuously from 0.8 to $2.5 \mathrm{THz}$ by lateral spatial chirping of the domain widths in the PPLN crystal and adjusting the sample temperature.
This work was supported by ARO under Grant No. DAAH04-96-1-0414 and by NSF under Grant DMR9729109.

${ }^{1}$ E. Knoesel, M. Bonn, J. Shan, and T. F. Heinz, Phys. Rev. Lett. 86, 340 (2001).

${ }^{2}$ C. Rønne, P. Åstrand, and S. R. Keiding, Phys. Rev. Lett. 82, 2888 (1999). ${ }^{3}$ C. Rønne, K. Jensby, B. J. Loughnane, J. Fourkas, O. F. Nielsen, and S. R. Keiding, J. Chem. Phys. 113, 3749 (2000).

${ }^{4}$ T. I. Jeon, D. Grischkowsky, A. K. Mukherjee, and R. Menon, Appl. Phys. Lett. 77, 2452 (2000).

${ }^{5}$ T. I. Jeon and D. Grischkowsky, Appl. Phys. Lett. 72, 3032 (1998).

${ }^{6}$ Y.-S. Lee, T. Meade, V. Perlin, H. Winful, T. B. Norris, and A. Galvanauskas, Appl. Phys. Lett. 76, 2505 (2000).

${ }^{7}$ Y.-S. Lee, T. Meade, M. DeCamp, T. B. Norris, and A. Galvanauskas, Appl. Phys. Lett. 77, 1244 (2000).

${ }^{8}$ T. B. Norris, Opt. Lett. 17, 1009 (1992).

${ }^{9}$ Q. Wu and X.-C. Zhang, Appl. Phys. Lett. 68, 1604 (1996).

${ }^{10}$ U. T. Schwartz and M. Maier, Phys. Rev. B 53, 5074 (1996).

${ }^{11}$ Handbook of Optics, 2nd ed., edited by M. Bass (McGraw-Hill, New York, 1995), Vol. 2.

${ }^{12}$ H. J. Baker, S. Hunsche, and H. Kurz, Phys. Rev. B 50, 914 (1994). 